\title{
A Conversation with Leonid Mirny
}

\author{
Interviewer: Beth Moorefield
}

\author{
Senior Editor, Nature Structural and Molecular Biology
} Leonid Mirny is a Professor at the Institute for Medical Engineering and Science and the
Department of Physics at the Massachusetts Institute of Technology.

Beth Moorefield: Your group has been elucidating the 3D organization of the genome using a combination of biophysical approaches to determine the dynamic properties of chromatin fibers that govern their folding and interactions, as well as Hi-C approaches to probe their structures and organization within the cell. Maybe we could talk about how the polymer models are actually helping to inform genome structure.

Dr. Mirny: My thinking about modeling in biology in general, and the role of modeling in chromosome biology, begins with realizing that models can test mechanistic hypothesis. If an experimental paper ends with a "Figure 4" and a hypothesis of how things work, a modeling paper starts with this hypothesis in Figure 1. The hypothesis of how it may happen is the starting point for our modeling; and experimental data are used to select and to validate the models. In our works on Chromosome Biology, we're not really building models of chromosomes based on Hi-C, instead we're using $\mathrm{Hi}-\mathrm{C}$ and other experimental data to test and select models that can best reproduce these experimental data.

The starting point is usually a hypothesis-maybe something that has been published, or maybe our own hypothesis of how we're thinking things may work - and then we test this hypothesis by putting suggested mechanism in the simulation box. For example, polymers should be of this length and have this type of interaction: Can this actually give rise to something that's observed experimentally? That's the overall framework of testing many possible models and finding those that can reproduce experimental observations. This follows my favorite Sherlock Holmes saying: "Once you eliminate the impossible, whatever remains, no matter how improbable, must be the truth."

Beth Moorefield: So basically, you're defining the parameters that then limit or constrict their movements or interactions?

Dr. Mirny: For example, parameters that control how different elements of the genome interact with each other or with DNA-bound proteins (e.g., cohesin or CTCF) or parameters of the chromatin fiber: diameter of the fiber, its flexibility, density of different chromatin fibers in the volume. If we cannot simulate a whole nucleus, we simulate a small volume where a few chromosomes or chromosomal regions interact. These are physical parameters. Plus, we add some biological interactions. We can say, "What if euchromatin is attracted to other euchromatic regions? Or what if heterochromatin is attracted to other heterochromatic regions. What's going to happen?" We run simulations forward and we see what happens.

For example, if we want to compare simulations with $\mathrm{Hi}$ $\mathrm{C}$ data, we compute frequencies of interactions between different regions. This gives us a simulated Hi-C map that we can then compare to the real Hi-C map. We usually sweep a broad range of parameters of the model and find a range of parameters where it agrees with $\mathrm{Hi}-\mathrm{C}$ data. If we see that it does not agree, we'll say, "Okay, we need to go back and revisit the assumptions of the model. Maybe it's actually heterochromatin that needs to interact with heterochromatin," or "We need to add some other interactions, for example interaction of heterochromatin with the nuclear lamina." That's our general approach.

Beth Moorefield: That approach actually has been revealing different levels of organization, but then also this reciprocal influence of chromatin fiber activity and structure.

Dr. Mirny: One of our main hypotheses now is that beyond pairwise interactions, active processes (i.e., ATP-consuming motors) are shaping genome structure. For example, loop extrusion is a process where the action of a molecular motor is shaping chromatin. Loop extrusion leads to formation of chromosomal domains, it can also facilitate and moderate enhancer-promoter interactions. But this requires a motor that can extrude loops, a motor that nobody has actually observed. That's why it's still a hypothesis. We believe that cohesin and other SMC [structural maintenance of chromosomes] proteins perform this particular mechanical function of being molecular motors that can extrude loops. We were lucky to establish collaborations with several experimental labs that started testing this model and the general biological roles of different components of the loop extrusion machinery, as well as other proteins that play a role in shaping chromatin architecture.

Beth Moorefield: Are these two parameters actually universal features of the organization of genomes, regardless of cell type or species?

(C) 2017 Mirny. This article is distributed under the terms of the Creative Commons Attribution-NonCommercial License, which permits reuse and redistribution, except for commercial purposes, provided that the original author and source are credited. 
Dr. Mirny: While specific parameters may differ, the process of loop extrusion can be rather universal. We see signatures of loop extrusion in eukaryotes as well as in bacteria. The most direct evidence of the loop extrusion mechanism actually comes from studies of bacteria: the work of David Rudner's group in Bacillus, and also works of Mike Laub's group in Caulobacter, who demonstrated that loop extrusion by SMC is essential for juxtaposition of chromosomal arms in bacteria and measured the speed of extrusion. We're focusing on mammalian systems and looking for evidence of similar mechanisms in other organisms. We and our collaborators have found that cohesin (another SMC protein) is needed for formation of domains in mouse cells, requiring one loop-extruding enzyme every few hundreds of kilobases of DNA, while, in S. cerevisiae, we and Jon Baxter's group found very low levels of loop extrusion. So, that's as far as we can go. As theoreticians we can say what's needed and what's not needed to reproduce the data. Occam's Razor logic would tell us that if you don't need it, it doesn't exist. But it's biology, so mechanisms can be very complicated... (shrugs)

Beth Moorefield: The size of the genome is going to affect how it's going to be packaged and then accessed. Do you find that there is strong relationship with size?

Dr. Mirny: The sizes of the genome and the nuclus certainly play major roles in shaping chromosomal organization. We and others have seen that in S. cerevisiae you can explain imaging and Hi-C data by just assuming that chromosomal arms are freely jointed chains. They are attached to the spindle pole body, but otherwise they're just free polymers, random walk polymers, and are sufficiently short and yeast nuclear volume is sufficiently large to accommodate uncompacted chromosomes.

At the same time, in mammal genomes, chromosomes are incredibly long and nuclear volumes are not enough to allow them to be open polymers, so chromosomes need to be compacted even during interphase, and this is certainly reflected in Hi-C data and microscopy. However, we noticed that in cells with larger nuclei, for example, mouse oocytes, chromosomes are much more open, almost like chromosomes in S. cerevisiae. We made this observation in our collaboration with the group of Kikuë TachibanaKonwalski in Vienna that did truly amazing single nuclei Hi-C. One of the interesting insights from the physics point of view is that when nuclei become much larger, chromosomes change their shape and organization. But they still have loop extrusion as evident from the presence of topological-associated domains there. So, mouse chromosomes even in very large nucleis are not really like yeast chromosomes.

Beth Moorefield: To always test the predictions of the physical models requires a lot of matching to the Hi-C data itself, which requires a lot of communication. What has been something that's actually helped to advance that kind of communication between the theoretical and the practical sides of these investigations?

Dr. Mirny: What really helped us is that we're not only developing models, but we're actually analyzing $\mathrm{Hi}-\mathrm{C}$ data from point zero, essentially from FASTQ files that come out of the sequencing machine. We decided to invest a lot of efforts in developing tools for processing and analysis of Hi-C data, for correcting various biases in Hi-C dataand this way we actually get first-hand experience with what's in the data - and also make sure that we're analyzing real signal rather than various artifacts that may have crawled into the data during processing. That also helps our communication with experimental colleagues, helps to develop a common language, common understanding. We constantly exchange ideas, brainstorm together. Again, our approach starts with a hypothesis. That's something where we certainly need lots of input from our colleagues on the biology side, who are closer to a specific biological system. From these interactions with our colleagues we also learned not to overgeneralize our findings from biophysical modeling; we try to avoid saying: "All chromosomes are like this, or all chromosomes are like that," because we understand that in different biological systems, the rules of the game can be very different. Nevertheless, we aim at finding sufficiently general physical principles and mechanisms that govern chromosome organization. And if we are lucky such mechanisms can be sufficiently universal; operate in different cell types, different organisms, and have multiple biological functions. We believe that loop extrusion is one of them. I'm very grateful to all the people who worked with us, particularly to Job Dekker, as well as Kikue Tachibana, Francois Spitz, Irina Solovei, and Bill Earnshaw, and the many others who were patient with us, helping and guiding us in developing models, analyzing and processing data, helping us develop intuition about chromosome organization in different biological systems.

Beth Moorefield: What would you say are some of the biggest advances to driving this field forward, in terms of working on the 3D genome organization?

Dr. Mirny: It may surprise you, but the biggest advance in this field, in my opinion, has become the progress in using archives for sharing papers prior to publication. bioRxiv and other archives have really accelerated the pace at which knowledge is disseminated. And now, practically everything appears on bioRxiv. I know from our own experience that it takes us a year or more to publish a paper. It's really unfortunate when you go to a conference and somebody tells you about some exciting findings, but it takes a year till you can read the paper and examine the figures when the paper is published. This has changed dramatically in the last year. Now, that practically everything goes into bioRxiv prior to publication we, as a field, are a year - if not more than a year - ahead of other fields that do not use bioRxiv. Other fields of biology may be behind, while physics and mathematics, have been using archives for decades.

I think it also changes the level of interactions between people. The field might become less competitive, in the negative connotation of competition. People go, "Okay, we're going to post this on bioRxiv; everybody's going to see it, we're going to get some feedback." I think it also improves the quality of publications because we get addi- 
tional feedback from colleagues who can suggest something, who can be critical, or praise your work. You really get a sense of where your work stands early on in the publication process. In the long run, I think it will accelerate other fields of biology because they'll have to catch up. If they depend on chromosome biology, they wouldn't want to be a year behind, so they will also start posting on bioRxiv.

Beth Moorefield: It gives another voice whose expertise is invaluable to critically evaluate the data, really.

Dr. Mirny: Exactly! But it's a bit more complicated when it comes to sharing data. At this point, bioRxiv only con- tains publications; people usually keep their data private until the date of the publication. In principle, there's no reason why everybody shouldn't release their data right away when they post a bioRxiv paper, but people don't want to be the first to do this. That's what happened with bioRxiv in the past: Initially, people were reluctant to post preprints, because they worried that this will give unfair advantage to people who don't post on bioRxiv. But if almost everybody's posting, you don't want to hold on to your paper and not post it; you want to catch up with everybody else. There is a critical transition, and I hope with data release the same will happen within the next year or so. 


\section{$\$_{\text {CSH\& }}^{\infty}$ Cold Spring Harbor Symposia SYMPOSIA}

\section{A Conversation with Leonid Mirny}

Cold Spring Harb Symp Quant Biol 2017 82: 403-405 originally published online May 4, 2018 Access the most recent version at doi:10.1101/sqb.2017.82.034850

Creative This article is distributed under the terms of the

Commons http://creativecommons.org/licenses/by-nc/4.0/, which permits reuse and

License redistribution, except for commercial purposes, provided that the original author and source are credited.

Email Alerting Receive free email alerts when new articles cite this article - sign up in Service the box at the top right corner of the article or click here. 